\title{
Managing Collaborative Innovation Networks - Practical Lessons from a Belgian Spatial Planning Initiative
}

\author{
Vidar Stevens \\ Department of Political Science, University of Antwerp, Antwerp, Belgium. \\ Annika Agger \\ Roskilde School of Governance, University of Roskilde, Roskilde, Denmark. \\ E-mail: vidar.stevens@uantwerpen.be
}

Received: July 21, 2017 Accepted: August 7, 2017 Online published: August 25, 2017 doi:10.5296/jpag.v7i3.11748

URL: https://doi.org/10.5296/jpag.v7i3.11748

\begin{abstract}
Collaborative innovation networks are increasingly used as vehicles for fostering innovative policy solutions. However, scholars have noted that the extent to which collaborative networks can actually contribute to the development of innovative policy solutions depends on how they are managed. Empirical research on the management of collaborative policy innovation processes is, however, scarce. Therefore, we review in this article a case to add new insights to the causal link between collaboration, management, and innovation. Specifically, we examine the management strategies which helped a Flemish administrative network to develop a radical new Spatial Planning Policy Plan. This study shows that the best way to manage collaborative innovation networks is not to press directly for results, but take the time to invest in relationship-building and together agree on a planning and clear process steps. Such a management approach allows actors to get to know each other and from thereon expand, with more background and appreciation for the others' goals, behaviors, and intentions, their group activities concerning the formulation of a radical and innovative policy plan.
\end{abstract}

Keywords: collaboration, innovation, management, governance, spatial planning.

\section{Introduction}

This article discusses how and if managers can facilitate collaborative processes of innovation. We understand management in this study as, "the endeavors and interventions of a central actor ('the manager') to facilitate collaborative networks, by shaping the conditions 
under which these networks operate and the involved stakeholders interact with each other" (Voets, Verhoest \& Molenveld, 2015: 983). Innovation, and more in particular 'policy innovation', is interpreted as, "creative search processes used to develop and realize new ideas and solutions that radically transform the way in which we are imagining and doing things in the public sector" (Ansell \& Torfing, 2014: 4). We specifically analyze the management of the collaborative process to develop a radically innovative Flemish Spatial Planning Policy Plan (FSSPP) in de Belgian NUTS 1 region of Flanders. In this case, government officials moved from the assumption that more concerted and innovative policy solutions will emerge for spatial planning problems, as more departmental organizations and thus more knowledge, resources, and experiences are included in the decision-making processes.

The focus on the management of collaborative processes to spur innovation in the public sector is very timely. In recent years, many Western governments have established collaborative arrangements, just like the Flemish government has done, to search for policy solutions that move beyond conventional wisdom and failed practices, and which are capable of getting a hold on the cross-cutting nature of many wicked policy issues, like immigration, global warming or coastal protection (OECD, 2014). In similar vein, scholars have in recent years also gained more interests in studying the causal link between collaboration and innovation in the public sector (e.g. Torfing, 2017; Stevens \& Verhoest, 2016a; Sørensen \& Torfing, 2012).

An often-heard finding in many of these scholarly works is that despite the highflying expectations in the innovative capacities of collaborative networks, the extent to which collaborations can actually contribute to the development of innovative policy solutions for cross-cutting policy problems depends on the way in which they are managed (Montin, Johansson \& Forsemalm, 2014). Participants of collaborations can, for example, hold different problem perceptions, may be reluctant to collaborate, or may paralyze the decision-making process for strategic reasons. Hence, management is necessary to get participants of collaborations moving in the same direction.

So far, the management strategies for exercising indirect control over collaborative processes have already been the subject of considerable research, most notably in the network management and governance literature (e.g. Huxham \& Vangen, 2005; Milward \& Provan, 2006; Agranoff, 2006; Koppenjan \& Klijn, 2004). Most of these studies focused on the management of a specific scenario of interdependency-driven network engagement; that is, the development of more or less the same kind of goods, services or policy solutions but then in an integrated manner.

Innovation, however, entails a clear break from the past, and thereby the radical transformation of existing and failed policy practices, ideas, and solutions (Sørensen \& Torfing, 2010). Or as Stevens \&Verhoest (2016a: 19) state, "within collaborative innovation processes, participants do upfront barely know what to expect; the only certainty they have is that the to be developed policy innovations (i.e. the innovative policy solutions) are meant to act a game-changers and radically alter the way in which a policy problem is addressed." For 
that reason, scholars have argued that the management of collaborative innovation processes is very different from the management of ordinary collaborations, as managers must not only steer for integrated results and compromises, but also foster creativity and out-of-the-box thinking among participants to develop radical new (not yet existing) policy paradigms, and manage the 'unknowns' (i.e. a not yet identifiable radical policy change in a specific context) that surround processes of innovation in the public sector (Bason, 2016).

Empirical research on the management of collaborative processes of innovation in the public sector has, however, remained scarce (Ansell \& Torfing, 2014: 238). This lack of research is striking because it means that we (i.e. 'the scientific community') do not fully understand how practitioners, in their role as managers, can live up to their potential in collaborative processes of innovation which take place in the public sector. In addition, it implies that we do not have a realistic sense of the value of collaborative networks as vehicles for the promotion of innovative policy solutions. Hence, the aim of this article is to get a more fine-grained understanding of what strategies and approaches managers apply at the micro-level in collaborative processes to spur innovation, and how network members experience these interventions and react thereupon. In this way, we can get a better grasp on the managerial drivers and barriers in such collaborative innovation arrangements.

For our data collection and analysis, we conducted interviews and a document analysis. The empirical data thus includes: relevant documents (e.g. meetings minutes, meeting agendas, parliamentary decrees, policy documents and position papers), and twelve transcribed interviews. Overall, this study shows that the management of collaborative innovation process is a complicated, slippery and sometimes even a plain unworkable exercise. When the manager is further confronted with a time pressure, the best way to manage these collaborative arrangements is not to press directly for results, but take the time to invest in relationship-building and together agree on a planning and clear process steps. Such a management approach allows actors to get to know each other and from thereon expand, with more background and appreciation for the others' goals, behaviors and intentions, their group activities concerning the creation of a radical and innovative policy change.

We continue as follows. The next section discusses more in-depth what we currently know about the management of collaborative processes of innovation in the public sector. Section 3 presents the case. Section 4 elaborates on our data and chosen methodology. Section 5 reports the case results, and section 6 - the final section - will reflect on the main findings of this study and put them in a broader perspective.

\section{The Art of Managing Collaborative Innovation Processes - Theoretical Perspectives}

In recent years, we have seen a gradual rise in the number of papers that focus on the management of innovation processes in collaborative networks (Ansell \& Gash, 2008). In many of these studies, scholars have argued that managers of these collaborative innovation processes do not 'command' in the same way as they might do in hierarchical organizations. The reason for this is that collaboration is typically voluntarily. In addition, as Bryson, Crosby \& Middleton Stone (2006) have indicated, collaboration operates in a 'shared power' world in which different stakeholders control specific resources and have their own distinct 
bases of power and authority. Hence, Ansell \& Gash (2013: 5) argue that the key adjective that can be used to describe the management of public sector innovation processes in collaborative networks is 'facilitative', i.e. managers may bear responsibility for steering colaborations toward efficient service delivery, consensus or creative problem solving, but they must work within the constraints imposed by voluntary action and shared power.

In specifying different management styles and strategies, scholars have mainly taken a contingency approach for explaining the behaviors of managers in collaborative networks. The contingency approach assumes that there is no single best way to exercise the management of collaborative networks; because, different tasks, goals, and contexts, place distinctive kinds of demands on managers (Agger \& Sørensen, 2016). In some collaborations, for example, the primary challenge of the manager may be to cultivate sufficient trust among the stakeholders in the collaborative network. In other situations, the core task of the manager can be to help an already functioning collaboration of stakeholders to be more creative or innovative.

Research on the collaborative processes of innovation and its management is, however, still in its infancy (Ansell \& Torfing, 2014: 238-239). Only a few scholars have offered accounts of possible strategies managers can use to ensure the development of policy innovations or innovative public services in collaborative innovation networks (see, for example, Bason, 2014; Stevens \& Verhoest, 2016a, Agger \& Sørensen, 2016, Keast \& Waterhouse, 2014).

Some of the findings and suggestions of these earlier studies are also quite contradictory to each other. A good example is a finding of Bason (2014: 220) in comparison to an outcome of the study of Keast \& Waterhouse (2014: 166). According to Bason, distortive management strategies (e.g. putting or even forcing organizations beyond their usual comfort zone) act as catalysts for creativity and innovation in collaborative networks. Keast \& Waterhouse, on the other hand, argue that integrative strategies, which are about encouraging and stimulating the genuine sharing of information among actors without any form of coercion, are most beneficial to spur idea generation in processes of policy innovation.

Nevertheless, the few conducted studies have increased our knowledge about the interactive dynamics and the management of processes of innovation in collaborative networks. The studies in the book of Ansell \& Torfing (2014), for example, provided us with more information about generative mechanisms between collaboration and innovation, and how these generative mechanisms can be fostered by a public leader or manager. In addition, we have gotten more knowledge about the determinants that hinder or stimulate learning among stakeholders at the micro-level in collaborative innovation networks and how a manager can spur learning among actors (Stevens, 2017).

Lastly, different taxonomies of management roles and tasks regarding the facilitation of collaborative innovation processes have been developed on the basis of single case studies. Agger \& Sørensen (2016: 5), for example, developed a taxonomy of management roles and tasks managers can perform to bring about 'collaborative advantage' for involved partners. More specifically, they argue that a manager of a collaborative innovation process must act as pilot, to give direction to the collaboration and keeping it on track, as whip to ensure that 
network members are not reluctant to participate in a collaborative manner in the innovation process, as culture-maker to normalize creativity and innovative behavior in the arrangement, and as communicator to spur dialogue in the collaborative arrangement and connect network partners. Other taxonomies are: the network management triangle (Stevens \& Verhoest, 2016a), the Innovative-Leadership Model of Termeer \& Nooteboom (2014), the four design attitudes of Bason (2014), and the Model of Facilitative Leadership of Ansell \& Gash (2012).

There are, however, still many empirical puzzles that need to be solved (see Ansell \& Torfing, 2014: 238-239), and variously accepted truisms which have to be empirically scrutinized (Stevens \& Verhoest, 2016b). For example, is it always the case that collaboration ensures that public innovation draws upon and brings into play all relevant innovation assets in terms of knowledge, imagination, creativity, courage, resources, transformative capacities and political authority (Sørensen \& Torfing, 2012: 5)? Moreover, are there different ways of collaboration that can stimulate and strengthen different aspects of the innovation process? And if so, what management is needed to support different forms of collaboration? Hence, we review a new case as a means to validate existing insights, and possibly increase our knowledge, about the causal link between collaboration, management, and innovation.

\section{The Flemish Collaborative Process to Develop an Innovative Spatial Planning Policy}

In this article, we focus on a Flemish administrative network consisting of twelve representatives from departmental organizations. The departmental organizations belonged to twelve different policy sectors ${ }^{1}$, as a means to capture the intertwined nature of spatial planning policies. The collaborative innovation network can be regarded as a top-down mandated administrative network, which political leaders permitted to develop a radical new policy strategy to better tackle problem issues within the field of spatial planning, like accessibility of infrastructure, cultural heritage preservation, social and economic cohesion, sustainable development, etc.

The administrative network was established in 2011 - but for this study we focus in on an 'innovation episode' between December, 2015 and February, 2016. During this innovation episode the administrative network members deliberately tried to develop a new Flemish Sustainable Spatial Planning Policy (FSSPP). The aim was not to generate more or less of the same kind of policy solutions, but rather to change the form, content and repertoire of policy actions, and even transform the underlying problem understandings, objectives, and program theory of the conventional governmental strategies (i.e. a radical transformation). This aim aligns with what Sørensen \& Torfing (2012) regard as an innovative policy change. For that reason, we perceive the aim of this process in the administrative network as a collaborative policy innovation process.

We believe that the case can be perceived as a most-likely case for collaborative processes to produce innovative policy outcomes, because many important enablers for collaborative innovation were present in the case, like: the support from the Flemish Minister President for the innovation process, the will of the involved departmental organizations to develop a

\footnotetext{
${ }^{1}$ The policy sectors in the administrative network were: education, welfare, enterprise, heritage, horizontal department of general affairs, mobility, spatial planning, economic affairs, ecology, employment, social affairs, and housing.
} 
creative and out-of-the-box policy plan, the freedom for the representatives in the administrative network to experiment with new policy ideas, and the involvement of organizational representatives who devoted more than fifty percent of their working time to the spatial planning project.

To this end, we expect that by examining the interplay between the behaviors of the network members and the strategies the manager of this Flemish administrative network utilized to spur collaboration and innovation, we learn more about what micro-level management strategies respectively enhance or slow down collaborative innovation processes, and thereby we get a better grasp on the managerial drivers and barriers in such collaborative innovation arrangements.

\section{The Methods to Study the Management Practices}

We used a case study methodology to study the management strategies of the manager of the administrative FSSPP network. We acknowledge the inherent limitations of using single case studies for extrapolating findings, as was described by George \& Bennett (2005). Yet, we also agree with Flyvbjerg (2011: 305) when he argues that a case study can further scientific development by the force of example. To this end, we do not pretend that our findings are fully generalizable or highly theoretical. However, we are convinced that our reflections are helpful in substantiating the development of theory on the impact of management on the innovative capacity of collaborative networks.

For the data collection, we drew on a detailed process mapping based on an analysis of documents and a series of interviews. The document analysis included: minutes of the meetings, position papers and policy documents of the representatives' organizations, (draft) versions of the final policy document, agendas of the meetings, and parliamentary decrees. The relevance of the documents was determined by making a selection based on whether the information in the documents said something new or extra about the different stagnations and breakthroughs of the policy innovation process, the various activities of the manager, and the positions of the actors with regard to the problem situation and possible solutions. The document analysis was complete once we reached data saturation.

Subsequently, the interviews helped us gain more insight into the behaviors, ideas, attitudes, and experiences of the members of the network with regard to the development of the collaborative innovation process, the interventions of the manager, and the process results. The interviews were semi-structured and the questions concentrated on the key events and insights that followed from the document analysis. In total, we interviewed all twelve representatives in the network (including the manager).

The interviews typically lasted an average of 1.5 hours. Each interview was recorded and transcribed. We guarenteed our respondents anonymity. Therefore, we numbered the interviews and used the phrase 'respondent (number)' to report quotes from the interviews in this article. We triangulated our interview data by comparing the interview responses to each other and to the document analysis findings. We followed-up with respondents if we ran into inconsistencies to ask for clarification. 
For the coding of both the document- and interview data, we used the NVivo software program. In this coding process, we made use of the earlier-discussed management roles (i.e. pilot, whip. culture-maker, and communicator) from Agger \& Sørensen's taxonomy (2016) as grounding concepts to code the documents and the interview transcripts. As such, this taxonomy allowed us to gain a notion of if the FSSPP network manager performed all different management roles during the collaborative policy innovation process and if so, which specific management strategies he utilized to perform these roles and for what reasons.

Subsequently, we coded the empirical data in such a way that we were able to distill how the different network members experienced and responded to these management strategies. Hence, in the results section we present how the manager of the administrative FSSPP network intervened in the collaborative innovation process, and how the network members experienced these managerial interventions and reacted thereupon.

\section{The Management Interventions in the Administrative FSSPP Network}

We start by presenting the results of the management activities of the manager as 'pilot'. As pilot a manager must give the overall direction to the collaborative innovation process (Agger \& Sørensen, 2016: 5). In the next few paragraphs, we thus describe what strategies the manager utilized, for what purposes, and with what effect, to keep the collaborative process on track.

\subsection{Pilot}

From the empirical data, it became clear that the role of the pilot was exercised by the manager. In giving direction to the collaboration and keeping it on track, the manager had to cope with two big challenges. First of all, the manager had to design the process in such a way that it was open and participatory. Secondly, because the political level expected a shared policy plan from the network members within two months', the manager had to make sure that the collaborative process did not take too long or become too messy (respondent 12).

To balance these challenges, the manager used a very 'controlling' management style (respondent 4). This controlling management style immediately started in the first meeting. After the standard introductions, the manager opened the discussion with what the final product should look like, what process steps would be followed, and what the schedule for the next weeks was. Furthermore, he demanded that all representatives cleared their agendas and devoted at least fifty percent of their time in the upcoming two months to the administrative collaboration. The manager argued that this amount of time was required for the individual representatives to read all their network peers proposals, to expand their knowledge about different types of spatial planning issues, and to organize a proper feedback loop with their home-organizations.

Respondents 8 and 10 argued that most representatives supported and understood the need for these controlling, or what they described as 'business-like', piloting interventions of the manager. Respondent 8, for example, explained: "at the start it was necessary to create a stable foundation from which we could work, otherwise it would have been difficult to make much progress in such a short period of time." 
However, there were also several points of critique by the network members. Respondents 3 , 4 and 6 all indicated that the high workload and short time planning of the project were very demanding; they felt they could not simply dedicate fifty per cent of their time to this spatial planning project, because they also had other work obligations. Consequently, especially respondent 4 constantly had the feeling that she was overtaken by events during the collaborative process. She had too little time to prepare for meetings, and she was unable to keep her home-organization's Minister and civil servants up-to-date on developments in the administrative network.

Other points of critique included the way in which the manager decided on what would be discussed in the general meetings and when. During the first meeting, the network members agreed that a shared agreement had to be reached within five meetings. Therefore, the manager urged the network members to ensure that they had their plans and proposals ready by the next session. The network members were only allowed to introduce a maximum of three policy proposals in the discussions. With this first 'selection mechanism', the discussions in the administrative network would remain containable, as it implied that only thirty-six policy proposals would be discussed in the collaborative arrangement.

Furthermore, to keep the final document readable and allow for a bigger impact on political discussion, the manager insisted that the final document would only include 10 to 15 policy proposals. So, another selection would occur in the course of the general meetings. Lastly, the manager unilaterally decided that each proposal would be discussed in the general meetings. However, if there was no general agreement on a proposal, or it did not appear that a shared agreement would be reached for that proposal in the next meeting, dialogue on that proposal would not continue.

These unilateral decisions of the manager about how proposals were to be selected and discussed frustrated many network partners. Respondent 6, for example, said that many representatives wondered whether the decision to institutionalize the 'selection mechanisms' was a legitimate act by the manager. In their opinion, this was the responsibility of the network members, who represented a certain organizational constituency, and not the call of the manager to make. Hence, to appease tempers the manager agreed with the organizational representatives, and in addition to the ten to fifteen main policy proposals, a thirty one-page appendix with the minority opinions and policy proposals would also be drafted.

This appendix helped the representatives show their Minister and senior administrative leaders that they "fought for their organizational interests" (respondent 2). Because, if a Minister or senior administrative leader merely saw the ten to fifteen main policy proposals, he or she would not get a full overview of the other discussions that took place in the collaborative arrangement (respondent 9).

Moreover, the way the manager organized the discussions and designed the decision-making process changed the way several network members behaved in the collaborative arrangement. For example, before the second meeting, the network members were asked to add their three policy proposals to an Excel spreadsheet. In this spreadsheet, they were also supposed to indicate whether they supported, were opposed, or had no opinion about others' proposals. 
Based on this initial scoring, it was decided whether or not a proposal would be discussed in the upcoming meetings. Therefore, because network members knew their proposals would be 'judged' by their peers prior to the actual discussions, they would strategically introduce proposals that would not find much opposition in the administrative network. Often, these were policy proposals that did not demand a radical policy change. Hence, respondent 2 argued, "this strategic selection of policy proposals reduced the innovative capacity of the collaborative arrangement." In addition, respondent 6 noted that many representatives "put their foot down' during the general discussion with the hope that this rigid behavior would increase the chance that their policy proposals would be included in the final policy document.

Looking back, the manager recognized that the process was not an easy linear process. Also, he knew that some network members felt that the innovative potential of the collaboration was not fully taken advantage of. Nevertheless, as a pilot, he was in a difficult balancing act between finding agreement and creating room for creativity and debate. Further, he had to perform this balancing act under time constraints. Therefore, he was very proud that after just two months he was able to present a document to the political level that had the support from all representatives' organizations.

\subsection{Whip}

Agger \& Sørensen (2016) describe the whip as a manager who ensures network members are not reluctant to participate in a collaborative manner in the innovation process. We have already noted that the manager was confronted with network members that showed rigid forms of behavior. Another challenge the manager had to deal with, as the whip, was the many 'new voices' around the table (respondent 4). New voices were network members who, up and until the case we are studying, had not been a member of the administrative FSSPP network. In contrast, the 'older network members' joined the administrative network in 2011 as representatives of their organizations.

Many of these new voices were relatively shy and waited a long time to see which way the cat jumps before they committed to any agreement in the collaborative arrangement (respondent 5). One of the 'shy' new voices reported the steep learning curve she experienced in the collaborative process (respondent 3 ). She noted that in the beginning, she found it very difficult to engage in the talks. She soon realized that all the other representatives acted as "lions who did their utmost to protect their organizational territory." Therefore, she also started to play the game more roughly, despite the feeling that this went against her human nature. She felt this was the only way to secure her home organization's interests in the collaboration.

To accommodate the participation of the 'new voices' in the network and to compensate for the influence of the 'shouters' in the collaborative arrangement, the manager used different strategies to stimulate what he called 'collaborative' behaviour (i.e. 'free, transparent and honest talking') among the representatives in the network. The type of strategy deployed depended on the specific behavior an individual used in the network. The manager tried to use a unique approach for each representative. 
Humor proved to be a powerful weapon to silence the 'dominant' representatives in the administrative network. By making a quip about someone's rigid behavior or rituals, the manager tried to let these dominant representatives know, in a clear but friendly manner, that "there were also other representatives involved in the deliberations (respondent 5)." As it was not the manager's intention to offend the representatives with his funny remarks, he used this management strategy sparingly and only used it on those who appreciated this form of communication. Alternatively, the manager would take a dominant or rigid representative aside 'in the corridors' to let him or her know that he did not appreciate their behavior in the general meetings (respondent 2).

For the 'introvert' representatives in the network, the manager had another method. In bilateral talks or conversations with a small number of other representatives, the manager tried to create 'safe-spaces' for introverts to help them feel comfortable sharing their opinions and thoughts (respondent 3). Another strategy was to directly ask the shy representatives their opinion on a specific matter during the general meetings (respondent 5).

Very occasionally, as a last resort, the manager would contact a very shy representative's senior leaders to inform them the interests of their organization were not well-represented in the interorganizational arrangement. The manager hoped that, with a little push from the home-organization, the shy representative would feel more pressure to actively engage in the discussions of the collaborative network.

According to many respondents, most of the time these management interventions, targeted at influencing representatives' behaviors, worked. Respondent 6 said, "that particularly after an intervention, she experienced as a shy person more space to make her points in the collaborative innovation network." However, after a short while, the representatives would intuitively return to their normal routines and habits (respondent 6). Hence, the manager's interventions did not appear to have a long lasting effect.

The manager, however, remarked that his primary task was not to silence people or drag information and ideas out of representatives' mouths. Ultimately, each individual was responsible for their own behavior and representation in the administrative network. Up front, the manager had already drawn up a code of conduct for how he expected the representatives to behave in the network. Specifically, this document stated that the representatives had to act transparently and reciprocally with their peers in the collaboration. This intervention was according to the manager sufficient steering from his side, because "the collaboration was not a kindergarten."

\subsection{Culture-maker}

In collaborative innovation networks, it is not enough for the manager to ensure that the network members behave in a collaborative manner. For a policy plan to become 'innovative', participants must use their imagination and creativity to develop solutions that go beyond conventional wisdom. Therefore, Agger \& Sørensen (2016) argued that a manager must also act as a culture-maker to normalize creativity and innovative behavior in the arrangement. However, in our case analysis, we only found two examples of interventions where the 
manager celebrated experimentation and creativity in the collaborative arrangement.

In the first instance, respondents 1, 4 and 11 indicated that at the start of every meeting, the manager emphasized the benefits of not only thinking about spatial planning from their 'organizational silos'. Instead, he encouraged the representatives to consider the contributions of others in the discussions with an appreciative point of view. According to respondent 4, "the great strength of the manager was that he encouraged us to search for new connections to solve spatial problems". He believed that new insights and beliefs could only follow from 'cross-fertilizations' between organizations (respondent 5). This motivated some organizations to find new synergies. For example, after years of indecision, evolving from this collaborative process, representatives from the Department of Cultural Heritage and the Agency of Economic Affairs developed new guidelines for the role of agricultural businesses in the preservation and restoration of monumental buildings.

The second example that we found was that the manager tried to break new ground by developing a shared language between the representatives. The manager noticed that during the discussions certain words seemed normal for some representatives, while they were jargon for others. The term 'quality of space' or 'mobility nodes', for example, had different meanings for network members (respondents 3 and 6). Therefore, at the end of each meeting, the manager added new words with definitions to a glossary. This way, the representatives created a shared interpretation of specific terms. The manager believed that, if they had more time, this glossary could have served as a roadmap to further develop new concepts or paradigms that better fit and defined the intertwined nature of spatial planning policies.

However, on the whole respondents 3, 5, 6 and 9 argued that creative solutions were seldom developed or discussed in the administrative network. It often seemed that representatives were not 'yet ready or prepared' to take risks; for example, they would not propose solutions that went beyond the instructions they got from their senior administrative leaders, cabinet members or ministers (respondent 3). Respondents attributed this to the feeling that there lacked a strong sense of commitment among the network members. According to respondent 9, "we barely knew each other, we had neither an idea of what the personal opinion of somebody was, nor a clue of how a person would respond to other opinions and criticisms in the administrative network."

Hence, respondent 2 suggested that it would have been helpful if the manager had invested more time and energy into cultivating a group mentality and developing a collective sense of possibility. Respondent 4 added, "the manager could have organized meetings at sea or in forests, just to get as far away as possible from politicians, senior administrative leaders and cabinet members of the Ministers." Respondent 4 believed that off-site meetings might have fostered an atmosphere where representatives were more comfortable sharing their personal views and opinions, and discussing creative policy solutions in a less politicized manner.

\subsection{Communicator}

The fourth and final, management role Agger \& Sørensen (2016) identified was the role of communicator. The communicator's job is to stimulate dialogue in the collaborative 
arrangement and connect network partners. The manager argues that he, in his role as communicator, was faced with a challenging interactive dynamics.

First of all, it was difficult connecting representatives who had opposing views on the origins of the spatial problems and possible solutions. Within the discussions, you could distinguish two major coalitions of representatives (respondent 12). These coalitions formed quickly based on similar ideas and solutions. The two coalitions held opposing views; they represented the basic tension of spatial planning policies between hard and soft interests - or as respondent 12 summarized it, "between stones and the natural use of the soil." Hence, the manager had to ensure that these two coalitions could find a middle ground which satisfied the needs of all the representatives' organizations which were a part of these coalitions.

Secondly, the manager said, "another thing you could notice in the discussions was that there were some bigger players in the collaborative network." Not bigger, in the sense, that these representatives felt more important than others, but that their peers gave their ideas more weight in the network discussions (respondent 3). In consequence, some representatives, like representative 4, often felt they were just "a little pawn in another person's or organization's game of chess." These representatives experienced a certain power imbalance in the collaborative arrangement. As such, the second challenge the manager encountered in his role as communicator was ensuring that the 'smaller' network members' ideas and concerns were also given enough attention in the discussions about the innovative policy change.

The manager used different management strategies to counter the perceived power imbalances and to cope with the basic tension between hard and soft spatial planning interests. In section 5.1 we elaborated on how the manager designed and structured the discussion- and decision-making process. The manager saw this management intervention as a hands-off strategy, implying that from the side-line he tried to develop decision-making structures so that certain interests would not prevail over others. Simultaneously, the manager was not afraid to use a more hands-on approach by joining and intervening in ongoing discussions. For example, many times the manager stopped discussions that ran aground, and he came back to them at a later point in time (respondent 6).

With regard to the first challenge, the manager did not attempt to 'break up' the coalitions of representatives. Instead, the manager was very lenient in allowing the two coalitions of representatives to have their own small groups discussions during the general meetings (respondent 2). In his view, these small group discussions could lead to breakthroughs if the clusters had enough room to get together and rethink their positions (respondent 5).

According to respondent 2, it was hard to say what would have happened if the manager had not allowed them to remain in their 'coalitions'. These coalitions were, besides building support for one's ideas, formed with the purpose to improve the positions of individual representatives as well as to steer the boat (read: the discussions) to a predetermined destination (respondent 2). This group formation was simply a part of the representatives' strategy. Hence, respondent 2 believed that if the manager had forced them to reconsider their coalition-building strategy they presumably would have abandoned the deliberations. 
However, when there were huge diametrical differences in points of view between the two coalitions, or specific members of the coalitions, but the manager had the feeling that a compromise could be reached if all parties lowered their swords, he was not afraid to urge the 'opposing' representatives to meet up in between meetings and present, if possible, a shared solution to the group in the next session. As such, the manager tried to place greater responsibility with the representatives who were 'upholding' the collaborative process (respondent 5).

To deal with the second challenge (that of the power imbalance in the administrative network), the manager used a dual strategy. First, the manager did not allow voting because "voting implies difference, for example, 5 people in favour and 6 people against." An agreement was thus reached if all network members, and thus also the 'smaller' representatives, agreed on a policy proposal. Second, the manager often summarized and reframed the discussions between 'powerful' network members in such a way that the ideas and suggestions of 'smaller' network members appeared to be the missing pieces of a grand puzzle.

According to respondent 4, the main effect of these management interventions was that representatives started looking for certain packaged deals and win-win solutions. For example, in the end, the two coalitions agreed on a package deal concerning the emergence of new economic activities in deprived rural areas.

During earlier meetings, both coalitions had already proclaimed that they recognized the urgency for the development of new economic activities in non-urban areas. Yet, for a long time they did not agree on whether the small scale producers had to be supported in these new economic activities by the government, or the bigger companies which could provide more labour and employment to the region. Eventually, with the help of the aforementioned managerial interventions, the network members realized that they already agreed on ninety percent of the issue; they simply disagreed on how to solve the problem. After this realization, it was just a small step to agree on a toolbox of measures which, in the view of many representatives, created the right balance between stimulating local entrepreneurship and attracting large-scale production companies to rural areas.

What helped the manager's efforts to facilitate dialogue and debate between representatives in the administrative FSSPP network, was that he was a civil servant from a horizontal department in the Flemish administration which directly fell under the supervision of the Prime Minister. This horizontal department was established with the purpose to facilitate transversal policy-making in Flanders. As such, the manager was not associated with any particular sectorial stakes or interests. His only job was to create unison between different sectorial interests on behalf of the Prime Minister. Respondent 1 reported that many representative saw the manager as a neutral authority who only wanted to develop the best solutions for spatial planning issues in Flanders.

To circumnavigate deadlocks, the manager sometimes got unexpected help from 'ordinary' representatives in the administrative network. Especially, about discussions on leisure- and sports activities and facilities, two representatives constantly played a brokering role in 
ameliorating differences between network partners (respondent 5). Specifically, these two representatives infused new narratives in the deliberations about how the development of recreational areas and business sites could go hand-in-hand, instead of leisure facilities being a compensation product for economic growth areas.

From our empirical data, it was hard to pinpoint whether these 'brokers' took on these roles for their own gain or for the benefit of the collective. The manager did assume that a shared agreement on these matters would also help the brokers develop their policy plans after the discussion on the FSSPP. Nonetheless, due to these interventions of these two ordinary representatives in the administrative network, it became easier for the manager to get the network members moving in the same direction on leisure and sports-related matters.

Retrospectively, respondent 6 argued that overall the manager's interventions as communicator could not change the reality that some representatives held positions in the administrative network where the involvement of their organization accrued more power within the collective. This was also not the main goal of the manager as a communicator. In the end, he mainly wanted to retain a reflexive gaze on the dynamics of the network, to promote effective interactions between the representatives.

\section{Discussion and Conclusion}

To conclude, research on the management of collaborative processes of innovation has been quite scarce. Therefore, we examined the management strategies of manager of a Flemish administrative network for facilitating the development of an innovative Spatial Planning Plan in the collaborative arrangement. We used Agger \& Sørensen's (2016) taxonomy of management roles as a heuristic to cluster the management strategies and to see what micro-level management interventions respectively enhanced or impeded the collaborative process of innovation in our case.

From our case analysis, it became clear that, despite the positive connotations of the word 'collaboration', in reality, collaborative innovation networks are decision-making arenas where organizational turfs collide. Management, as such, can help to achieve unison and foster the development of innovative policy solutions for complex, intertwined policy problems, like issues of spatial planning. At the same time, in our case, the management itself proved to be a complicated, slippery and sometimes even a plain unworkable exercise. Overall, we believe that on the basis of our case results for each management role of the taxonomy of Agger \&Sørensen (2016) a lesson can be drawn regarding how a manager can foster the development of innovative policy solutions in collaborative innovation networks.

The first lesson follows from the way in which the manager of the FSSPP network, in its role as pilot, structured and designed the decision-making and selection process. Specifically, the manager decided to work in two months' time towards a policy document which would have the support of all representatives' organizations and only include ten to fifteen new policy solutions. To achieve this, the manager followed a very strict decision-making and selection procedure. As a result, the representatives experienced that they only had a few opportunities to leave their mark on the new policy plan. Moreover, they were well aware of the fact that if 
they proposed policy proposals that required too much of a radical policy change, their chances of success (in terms of getting the support from other representatives' organizations) would decrease. Therefore, they mainly proposed solutions that only demanded a marginal adoption of existing policies. In addition, they showed a lot of foot-draggings to ensure that they were eventually not the ones that compromised on their chosen positions.

Hence, on the basis of these case dynamics, we advise managers in their piloting activities not to be overly controlling in steering the decision-making process. In addition, the 'piloting manager' should create sufficient decision-making opportunities for the network members to leave their mark on the (to be developed) innovative policy plan. Otherwise, the network members will start acting strategically and primarily look at their own policy interests. In consequence, the decision-making process will turn into a bargaining and negotiation process, instead of a creative endeavor between involved participants; causing that the newly developed policy solutions tend to become mere marginal adoptions of existing policies, rather than radical new, out - of -box, policy solutions.

This first lesson has some resonance in the collaborative innovation literature. Stevens \& Verhoest (2016a) and Agger \& Sørensen (2016) respectively argue that the potential for innovation increases if the manager uses a more laissez-faire approach in facilitating the decision-making process. Such an approach places greater responsibility on the network members and makes them more an owner of the collaborative innovation process, instead of a mere individual that is 'allowed' to participate in the process. The network members thus become more involved, which, in turn, increases their commitment to the development of innovative policy solutions.

For the management role of 'whip' the following important lesson stands out in our case study: if the manager does not see it as his or her primary task to direct interventions at changing actor behavior, these managerial efforts will only have a temporal effect, and the concerns and opinions of the 'shouters' will prevail in the collaborative arrangement. In our case, the manager, in its role as whip, utilized for both introvert as well as dominant representatives different strategies to accommodate their participation in the collaborative arrangement. The effects of these interventions were most noticeable just after the intervention took place, as introverts felt more space to articulate their concerns. Yet, after a short while, the representatives felt back in their routine behaviors. The managerial interventions targeted at changing actors' behaviors thus had no long lasting effect.

Although, the manager wanted to promote collaborative behavior (i.e. free, transparent and honest talking) among the members in the collaborative innovation network, he did not perceive himself as the manager who pulls information out of introvert people or silences the more dominant representatives. In addition, the collaborative innovation process was in his view not a kindergarten and, therefore, he held the opinion that every representative was in the end responsible for its own way of representing and behaving.

Psychological studies point, however, that behavioral changes do not occur overnight or just by a simple (short-term) intervention by a manager. Instead, for each behavioral change, a person has to go through a process starting from precontemplation (i.e. reacting negatively by 
reflex if we receive ideas from others that we have to change our behaviors), to acceptance, action, and maintenance of a new way of behaving (Prochaska \& DiClemente, 1983). To avoid that individuals revert to their negative routine behaviors, they need for a longer period of time guidance and support.

If we relate these suggestions from the psychological literature to the management of behaviors of individuals in collaborative processes of innovation, it is recommended that network managers, in their role as 'whip', really see it as their core duty to cultivate and support, for a longer period of time, behavioral changes among network members for the benefit of the interactive dynamics in collaborative innovation networks. This is, of course, only possible if the manager has sufficient time and can find a way to create conditions that will allow those who are willing to change, to reveal their personal difficulties, experiences, and emotions, and to take the steps necessary to solve their behavioral discomfort.

A third important lesson that follows from our case study is that members of collaborative innovation networks first need to get to know each other before they are actually willing, and most of all capable, to develop innovative policy solutions that move beyond conventional wisdom and practices. This lesson stresses the importance of the manager as 'culture-maker' in the collaborative innovation process.

In our case, we saw that many representatives were foremost busy with what their own organizations could gain from the collaboration. Just like Huxham \& Vangen (2005), however, we believe that a real collaborative advantage (e.g. access to new resources, cost-efficiency, or, like in our case, comprehensive innovative policy solutions) can only be created if representatives do not hold on too much to their own organizational instructions and priorities, but instead also take some risk by seeking for more convergence with their partners' goals and interests.

What was mainly holding representatives back from having a more open attitude and appreciation towards the ideas and interests of other representatives' organizations, was a lack of awareness about 'the other'. The representatives neither knew each other very well nor had they much knowledge about what representatives' personal ideas and opinions were or how he or she might respond to criticisms by peers in the administrative network. In addition, more than half of new members were 'new voices' around the table who were not familiar with how 'normally' idea-generation and -development took place in the administrative network.

Therefore, we recommend managers of collaborative innovation networks, in their role as culture-makers, to not directly press for results but take the time to invest in relationship-building, when at the start of the innovation process there are no strong bonds or working relationships between network members. Such a management approach allows network members to get to know each other, and from thereon expand, with more background about the others' goals, behaviors, and intentions, their activities concerning the creation of a radical and innovative policy change.

This third lesson has some similarities with what Bason (2014: 222) describes as the strategy 
of managing discomfort. According to Bason (2014: 222), one of the biggest challenges for managers of collaborative innovation networks is to get actors 'surrender' to a situation in which they have to explore a problem space with others and search for yet unknown innovative solutions. Bason also recognizes that particularly at the beginning of an innovation process actors are faced with a lot of uncertainty about the behavior and intentions of others with whom they have to collaborate. Therefore, he (2014: 223) argues that a manager should take sufficient time to help involved representatives and their organizations to get used to the novel situation and adapt to the 'unknowns' of the collaborative innovation process.

However, in regard to Bason's statement, we do want to make the important disclaimer that the concept of 'sufficient time' should not be used as an excuse to delay the collaborative innovation process. In the end, 'innovation' entails a clear break from past practices and not exactly knowing how these policy changes eventually affect the practices and responsibilities of all involved organizations. As such, we do also agree with Michlewski (2008) when he argues that not all uncertainty and behavior can be managed and at a certain point the manager and involved individuals should simply embrace the cooperation in order to get things going.

The fourth, and final, lesson that emerges from the case study analysis relates to the management role of 'communicator'. Communicators must be aware that individuals participating in collaborative innovation processes intrinsically, perhaps, see the need to innovate, but as a representative, they are also driven by other motives, like the need to 'win for their organization' in a collaborative process instead of losing. Therefore, a communicator must not demand representatives to leave their individual (organizational) agendas and expectations at the door but instead work with these strategic motives when connecting different parties in the collaborative arrangement.

In our case, many representatives joined actor coalitions to find support for their individual ideas, to strengthen their positions, and to steer the discussions in a predetermined position. The manager did not break up these coalitions or insist that individual representatives stepped beyond their organizational interests. Only when there were diametrical differences, but the manager believed that a compromise could be reached, he intervened and urged the 'opponents' to find common ground on a policy proposal. In consequence, many package deals were made during the collaborative innovation process which satisfied the needs of most representatives of the coalitions. Sometimes, the manager even got help from representatives who acted as brokerages to catalyze the development of package-deals between opposing coalitions.

Hence, these interactive dynamics teaches us that the natural realities of collaboration are oftentimes the opposite of what we pretend them to be. In reality, each representative around the table carries with her or him the expectations, instructions, pressures, and politics of their home-organizations, and he or she will adapt their network behaviors to these factors. Yet, by working with these individual agendas and placing greater responsibility with the representatives, these individual agendas can through coalition-formation, bargaining and negotiation (or, even other forms of interactions) be turned into package deals and shared 
goals which, in turn, can spur the development of comprehensive innovative policy strategies.

Of course, this research also has certain limitations. In the article, we already addressed the small-N problem of a single case study, and thereby the issue of context-dependent generalizations. Additionally, because we only studied the management of a collaborative innovation process where the network members eventually achieved to develop a policy plan, but we did not compare our findings to a case where the actors with the help of a manager did not agree on an innovative policy solution, our results and final reflections may have some bias. In consequence, the micro-level managerial approaches that we suggest to be most beneficial given the complex institutional dynamics of our collaborative innovation network, can have a smaller positive influence on the interactions between network members than we proclaim.

Therefore, we, first of all, propose that prospective studies examine the management of collaborative networks where the involved actors did not succeed in agreeing on innovative policy solutions. Such an analysis would verify or falsify our research findings for the different management roles. Secondly, we encourage scholars to also look at other complex innovation- and governance contexts. It would, for example, be interesting to see how the management dynamics for the promotion of policy innovations in collaborative networks that operate in multi-level governance structures differ from our case findings. In this way, we believe that the research niche of the management of collaborative innovation networks can further mature, and thereby enrich the scholarly debates on how management can spur collaborative innovation in the public sector.

\section{Acknowledgments}

This work was supported by the Belgian Science Policy under grant BR/132/A4/BRAIN-TRAINS.

\section{References}

Agger, A. \& Sørensen, E. (2016). Managing Collaborative Innovation in Public Bureaucracies. Planning Theory. https://doi.org/10.1177/1473095216672500.

Agranoff, R. (2006). Inside Collaborative Networks: Ten Lessons for Public Managers. Public Administration Review,66 (special issue): 56-65.

Ansell, C., \& A. Gash. (2008). Collaborative Governance in Theory and Practice. Journal of Public Administration Research and Theory, 18(4), 543-571.

Ansell, C., \& A. Gash. (2012). Stewards, mediators and catalysts: Toward a Model of Collaborative Leadership. Innovation Journal: The Public Sector Innovation Journal, 17(1), $1-21$.

Ansell, C., \& Torfing, J. (2014). Collaboration and design: new tools for public innovation. This is a chapter. In C.Ansell \& J. Torfing (Eds.), Public Innovation through Collaboration and Design (pp. 1-19). New York, N.Y.: Routledge.

Ansell, C., \& Torfing, J. (2014). Collaborating on design - designing collaboration. This is a 
chapter. In C.Ansell \& J. Torfing (Eds.), Public Innovation through Collaboration and Design (pp. 229-239). New York, N.Y.: Routledge.

Bason, C. (2014). Design attitude as an innovation catalyst. This is a chapter. In C.Ansell \& J. Torfing (Eds.), Public Innovation through Collaboration and Design (pp. 209-229). New York, N.Y.: Routledge.

Bryson, J. M., Crosby, B. C., \& Middleton Stone, M. (2006). The Design and Implementation of Cross-Sector Collaborations: Propositions from the Literature. Public Administration Review, 66(special issues): 44-55.

Flyvbjerg, B. (2011). Case study. This is a Chapter. In N.K. Denzin, N. K. \& Y.S. Lincoln (Eds.). The Sage Handbook of Qualitative Research. Thousand Oaks, CA: Sage.

George, A. L., \& Bennett, A. (2005). Case studies and theory development in the Social Sciences. Cambrige, MA: MIT Press.

Huxman, C., \&Vangen, S. (2005). Managing to Collaborate; The Theory and Practice of Collaborative Advantages. London: Routledge.

Montin, S., Johansson, M., \& Forsemalm, J. (2014). Understanding innovative regional collaboration: metagovernance and boundary objects as mechanisms. This is a chapter. In C. Ansell \& J. Torfing (Eds.). Public Innovation through Collaboration and Design (pp. 106-125). New York, N.Y.: Routledge.

Keast, R., \& Waterhouse, J. (2014). Collaborative networks and innovation: the negotiation-management nexus. This is a chapter. In C. Ansell \& J. Torfing (Eds.). Public Innovation through Collaboration and Design (pp. 148-170). New York, N.Y.: Routledge.

Klijn, E. H., \& Koppenjan, J. (2004). Managing Uncertainties in Networks. New York: Routledge.

Milward, H. B., \& Provan, K. G. (2006). A Manager's Guide to Choosing and Using Collaborative Networks. Retrieved from the IBM Center for the Business of Government website: http://www.srpc.ca/ess2016/summit/Reference_9-Milner.pdf.

OECD. (2014, November). Innovating the Public Sector; From Ideas to Impact. Paper presented at the OECD Conference, Paris, France.

Prochaska, J., \& DiClemente, C. (1983). Stages and processes of self-change in smoking: toward an integrative model of change. Journal of Consulting and Clinical Psychology, 51(3), 390-395.

Sørensen, E., \& Torfing, J. (2010). Collaborative Innovation in the Public Sector: an analytical framework. Ritsumeikan Hogaku, 330(2), 209-236.

Sørensen, E., \& Torfing, J. (2012). Enhancing collaborative innovation in the public sector. Administration and Society, 43(8), 842-868.

Stevens, V. (2017, in press). Individual learning behaviour in collaborative networks. This is a 


\section{Macrothink}

Journal of Public Administration and Governance

ISSN 2161-7104 2017, Vol. 7, No. 3

Chapter. In C. Dunlop, C. Radaelli, \& P. Trein, Learning in Public Policy: Analysis, Modes and Outcomes. Palgrave-MacMillan, Basingstoke, United Kingdom.

Stevens, V., \& Verhoest, K. (2016a). How to Metagovern Collaborative Networks for the Promotion of Policy Innovations in a Dualistic Federal System?. The Innovation Journal: The Public Sector Innovation Journal, 21(2), 1-26.

Stevens, V., \& Verhoest, K. (2016b). A Next Step in Collaborative Policy Innovation Research: Analysing Interactions using Exponential Random Graph Modelling. The Innovation Journal: The Public Sector Innovation Journal, 21(2), 1-20.

Termeer, C., \& Nooteboom, S. (2014). Innovative leadership through networks. This is a chapter. In C. Ansell \& J. Torfing (Eds.). Public Innovation through Collaboration and Design (pp. 170-188). New York, N.Y.: Routledge.

Torfing, J. (2017). Collaborative Innovation in the Public Sector.Georgetown University Press.

Voets, J., Verhoest, K., \& Molenveld, A. (2015). Coordinating for Integrated Youth Care: The need for smart metagovernance. Public Management Review, 17(7), 1-21.

\section{Copyright Disclaimer}

Copyright for this article is retained by the author(s), with first publication rights granted to the journal.

This is an open-access article distributed under the terms and conditions of the Creative Commons Attribution license (http://creativecommons.org/licenses/by/4.0/). 